Relation between changes in posteroanterior stiffness and active range of movement of the cervical spine following manual therapy treatment

Neil Tuttle, MPhil

Rod Barrett, PhD

Liisa Laakso, $\mathrm{PhD}$

School of Physiotherapy and Exercise Science, Griffith University, Gold Coast Campus

Address for Correspondence or reprints:

Neil Tuttle

School of Physiotherapy and Exercise Science

Gold Coast campus, Griffith University

Queensland, Australia 4222

Phone: +61 755528930

Fax: +61 755528674

Email: $\underline{\text { n.tuttle@griffith.edu.au }}$

This is a non-final version of an article published in final form in Spine Volume 33 - Issue 19, pp E673-E679. 


\section{Mini Abstract/Précis.}

Correlations between changes in posteroanterior stiffness at symptomatic locations of the cervical spine and changes in range of movement following treatment demonstrate stiffness of passive posteroanterior movements is related to active range of movement and suggest the therapeutic effect of manual therapy treatment is localized to the symptomatic location. 


\section{Background}

2 Posteroanterior (PA) movements are one form of motion palpation commonly used by manual therapists to assess and treat spinal pain and are produced by applying a force over a vertebra (Figure 1). Although originally thought to produce, and thus enable the clinician to assess, a translational gliding movement of one vertebra on another ${ }^{11}$, it is now clear that the spinal movement produced by PA movements is neither localized to one intervertebral level nor predominantly translational ${ }^{26}$. Nevertheless, according to the manual therapy paradigm PA movements are still intended to assess the mobility of the underlying intervertebral segment which in turn is thought to be related to impairments such as pain and limitation of active range of movement (AROM) ${ }^{21,24}$. A variety of subjective descriptors are used to represent the findings on manual assessment of PA movements but there is limited agreement on the meaning of even the most common descriptors ${ }^{22}$. Maitland ${ }^{23}$ advocated the use of subjectively referenced force displacement curves known as movement diagrams as a means to describe and communicate findings from manual motion testing, but the validity of movement diagrams has been brought into question ${ }^{28}$. More recently objective measures of The presumed relation within the manual therapy paradigm of PA movements to underlying intervertebral movement is supported by a limited number of studies. In an in vitro study of the thoracic spine, Sran et al. ${ }^{34}$ found increased PA stiffness corresponded to increased local intervertebral stiffness while Kawchuk et al. ${ }^{16}$ using a porcine model reported increased PA stiffness with artificially induced disc degeneration. There is some limited support for a relation between PA stiffness and patient impairments with one study reporting changes in PA stiffness being related to changes in pain and AROM in the lumbar spine ${ }^{19}$. Altered PA stiffness as assessed in these studies however does not appear to be specific to local or intervertebral factors. Differences in PA stiffness have also been found with factors whose effect would be expected to extend beyond local or intervertebral movement such as patient position ${ }^{7,9}$ stage of respiration ${ }^{31}$, direction of movement ${ }^{5,2}$, indentor size ${ }^{33}$, rate of movement 
1 Most studies of PA movement have focused on the lumbar spine ${ }^{30}$ and used only single

2 values of displacement or linear approximations of stiffness to characterize PA movements. It

3 has recently been suggested that relations between applied force and displacement during PA movements are non-linear ${ }^{27}$. For example in the cervical spine, differences were not found between tender, potentially symptomatic ${ }^{15}$ locations and less tender locations in either displacement or single values of PA stiffness. Differences were however detected when nonlinear methods such as simultaneous confidence bands (SCB) and simultaneous prediction bands were used to assess differences in PA stiffness ${ }^{38}$. This analysis however was also not sufficient to specifically characterize the differences in PA stiffness between sides.

Just as PA movements are intended to assess local stiffness, when used as treatment techniques PA movements are thought to improve impairments by decreasing local stiffness ${ }^{20}$. A relation between PA movements and impairments could be inferred if characteristics of PA movements change when impairments change. Furthermore, if a relation between PA movements and impairments only occurs for specific locations of PA movements and only following treatment directed towards those particular locations, it could be inferred that the therapeutic effect of the treatment is localized and specific to the treated location. A strategy for clinical reasoning advocated by Maitland ${ }^{23}$ and commonly employed in clinical practice ${ }^{12}$ uses changes in active movements immediately following treatment as an indicator of change in impairments. The advantages of investigating changes immediately following treatment are that this timing corresponds to the timing of reassessments in clinical practice. In addition the short time between reassessing PA movements and AROM would be expected to maximize the likelihood of detecting relevant relationships by minimizing the impact of extraneous factors on measures of both AROM ${ }^{14}$ and PA movements ${ }^{37}$. Furthermore, AROM is one of the few measures of impairments that not only is known to change immediately following treatment ${ }^{27}$ but also has been shown to be a predictor of longer-term changes in patient symptoms ${ }^{39}$.

An aim of the current study was to determine if differences in AROM and PA stiffness following treatment by PA movements to the cervical spine are dependent on the treated location. A second and more important aim was to determine if there is a relation between 
1 improvement in AROM and reduction in PA stiffness. It was hypothesized that following

2 treatment by PA movements to locations deemed to be symptomatic there would be: 1) an

3 increase in AROM; 2) a decrease in PA stiffness at the treated location; and 3) a significant

$4 \quad$ relation between decreased PA stiffness and increased AROM. It was expected that an

5 increased understanding of the relations between PA movements and patient impairments

6 resulting from this study would contribute to a greater understanding of musculoskeletal neck

7 pain and its treatment by manual therapy.

$9 \quad$ Method

10 Subjects

11 Subjects were recruited by a broadcast email to all staff and students at a university. The

12 inclusion criteria were neck pain for more than 2 weeks and a reported limitation of active

13 neck movements. Exclusion criteria were a current third party claim, a history of trauma

14 within the previous six months, or any contraindications to treatment by manual therapy ${ }^{24}$.

15 Note insert page number twenty subjects (10 females and 10 males) with a median age of 31

16 years (range $19-55$ years) who met the criteria volunteered to participate in the study which

17 took place in May and June 2007. The median symptom duration was 2 years (range 3 weeks -

1820 years). Over the previous three days, the reported average pain intensity was 3/10 (range

$190.5 / 10-7 / 10$ ) and maximum pain intensity was 4/10 (range 1/10 - 10/10). Investigator A was

20 a titled Musculoskeletal Physiotherapist with over 30 years experience and Investigator B was

21 a registered physiotherapist with 2 years experience.

Design

24 After providing written confirmation of informed consent, each subject attended a single test 25 session lasting approximately ninety minutes, Investigator A took the patient's history and 26 performed a physical assessment including active movements and passive motion palpation.

27 Based on his assessment, Investigator A selected two locations between C3 and C6. One 28 location that was assessed as being hypomobile and considered most likely to contribute to the 29 patient's impairments was deemed symptomatic. A second location deemed to be 30 asymptomatic was selected on the opposite side either $12 \mathrm{~mm}$ (approximately one 31 intervertebral level) above or below the symptomatic location depending on which location 
1 was considered less likely to be a significant contributor to the patient's impairments. The 2 symptomatic location was located along the articular pillar, but the height was not defined by a 3 specific anatomical location. Many authors suggest that unilateral PA forces should be applied 4 over the apophyseal joints, but this was not specified in this study for two main reasons.

5 Firstly, anatomic locations cannot be located accurately without imaging. Perhaps more 6 importantly, the apophyseal joints may not be the most appropriate location for applying PA forces. The intervertebral movements produced by PA movements are known to be primarily 8 extension and the axes of rotation of cervical extension generally go through the vertebral 9 body of the lower level of a motion segment rather than through the apophyseal joints ${ }^{4}$. Even 10 if the location of the application of PA force were to be defined anatomically, it may therefore 11 be more appropriate to do so in relation to centres of rotation rather than apophyseal joints.

12 Investigator B was informed of the locations, but not which site was symptomatic or 13 asymptomatic.

Investigator B assessed AROM and PA stiffness. The subject remained on the treatment bed for a further two minutes. Investigator A performed one of three experimental interventions (standard, placebo or control) for two minutes and the subject remained prone for an additional two minutes. Measures of skin conductance, skin temperature and blood flux were taken during this time, but are not reported in this paper. Investigator B then reassessed AROM and PA movements. The post-intervention assessments formed a baseline for the subsequent intervention. The order in which the first three interventions were performed was determined prior to the first intervention by Investigator A selecting one of 20 pre-printed forms with a random intervention order. The process was repeated for the next two interventions. AROM and PA stiffness at the two locations were measured before and after each of four interventions: 1) a standard intervention consisting of two-minutes of PA mobilization to the symptomatic location as would be performed if the subject were to receive that technique as a clinical treatment; 2) a placebo intervention consisting of the same grade of PA mobilization as on the symptomatic location but performed at the asymptomatic location; 3) a control intervention where the subject simply remained in the same position for two minutes; and 4) a general treatment intervention that consisted of manual therapy (not including high velocity thrusts) to the cervical spine as might occur in a normal treatment 
1 setting and not limited in duration or to specific techniques or locations. The general treatment

2 intervention was not restricted to treatment techniques performed in supine so was always the

3 last to be performed thereby precluding blinding of Investigator B to this intervention.

Data from a previous study ${ }^{38}$ was used to conduct a power analysis that indicated a sample size of 18 would provide a power of 0.95 .

Instrumentation and data collection protocol

The Posteroanterior Movement Assessment Device (PMAD) mounted on a re-positionable frame was developed to assess unilateral PA movements of the cervical spine in a manner as similar as possible to manual palpation (Figure 1). When using the device, an indentor is directed manually along a linear path and measurements of the applied force and the resulting displacement are recorded. Five movements were performed at a frequency of approximately $1 \mathrm{~Hz}$ and a single set of force and displacement data was calculated from three of the final four movements. As the entire FD curves from the PMAD were to be considered, repeatability was assessed using coefficients of multiple determination (CMD). The CMD was 0.90 for intraday, intra-rater repeated measures as used in the current study, but on an asymptomatic population. A more complete description of the methodology and repeatability is presented elsewhere ${ }^{37}$. It was necessary to ensure that when PA movements were produced by the operator's thumb such as during manual palpation, the standard intervention and the placebo intervention, that the movements occurred in the same planes as the PA movements measured by the PMAD. The movement of the operator's thumb was therefore constrained during these procedures by a gating mechanism attached to the frame of the PMAD (Figure 1).

A head-mounted 3-axis orientation sensor (3DM MicroStrain Inc, 310 Hurricane Lane, Williston, VT, USA) was used to measure AROM. The patient was seated in a high-backed unpadded chair with his or her shoulders against the backrest and their arms by their side holding the top of the rear chair legs. Investigator B demonstrated the movements to be performed and after zeroing the sensor, the patient was asked to perform three movements "as 
1 far as you can reasonably move" in each cardinal direction ${ }^{36}$. The movements were

2 unconstrained and performed at the patient's selected speed as would typically occur in the

3 clinical setting. The maximum of the three movements in each direction was used for further

4 analysis. Full axis movements have been demonstrated to be more repeatable than half axis

5 movements ${ }^{18}$ so full axis movements of flexion/extension (FE), lateral flexion (LF) and

6 rotation (ROT) as well as the total range of movement about all three axes (TROM) were used

7 for further analysis. The difference between repeated measures was the variable of interest, so

8 repeatability had been assessed using limits of agreement ${ }^{3}$. For asymptomatic subjects, the

$995 \%$ limits of agreement were from -5.9 degrees to 3.5 degrees ${ }^{36}$.

Data and statistical analysis

12 Stiffness values for PA movements were interpolated to produce 100 data points at $0.25 \mathrm{~N}$

13 force intervals from 0.5 to $25 \mathrm{~N}$ and the percentage changes in PA stiffness from pre-

14 intervention to post-intervention were calculated. A bootstrap re-sampling procedure ${ }^{38}$ was

15 used to calculate one-tailed 90\% SCBs for differences in PA stiffness following each

16 intervention for each side. Levels of force where the SCBs of the differences did not include

17 zero indicated regions where PA stiffness was likely to have reduced. Continuous regions of

18 the SCBs of the differences for the standard intervention that included, and did not include zero were considered separately for further analysis. The primary reason for determining regions where changes in stiffness were likely to have occurred was for use in further analysis. Rather than dividing the data into arbitrary regions, it was considered that if changes in AROM were related to changes in PA stiffness, such relations would be more likely to occur in regions where PA stiffness changed in response to the standard intervention. As a result a 90\% criteria was used for determining the SCBs rather than a usual, more stringent 95\% criteria. Repeated measures ANOVA were used to assess within subject changes by intervention for AROM and regional PA stiffness. Type 3 sum of squares, uncorrected pairwise contrasts were performed for within subject differences across interventions. Pearson correlation coefficients were calculated between change in TROM and change in mean PA stiffness for both the symptomatic and asymptomatic sides following each intervention. Any significant correlations were explored by calculating correlation coefficients between TROM 
1 and each region of PA stiffness and between each axis of AROM and the mean PA stiffness.

2 Significance levels for ANOVAs and correlation coefficients were set at $p<0.05$.

\section{Results}

5 Representative stiffness curves for the standard intervention for two subjects showing pre-

6 intervention, post-intervention and differences in stiffness are shown in Figure 2. Mean initial AROM (SD) for FE, LF, ROT and TROM in degrees were: FE = 119 (17); LF = 93 (12); ROT

$8=137$ (16); and TROM = 349 (39). The initial stiffness for each side and region of stiffness

9 are shown in Table 1. The SCBs of percentage changes in PA stiffness following the standard

10 intervention are shown in Figure 3. The two regions of PA stiffness at forces where the SCBs

11 did not include zero (4-8 $\mathrm{N}$ and 14-18 $\mathrm{N}$ ) as well as two regions that included zero (8-14 N

12 and 18-25 N) were used for further analysis. The lower boundaries of the SCBs were greater

13 than zero for their entire length for the asymptomatic side and following all other

14 interventions.

[Insert Table 1 about here]

[Insert Figures $2 \& 3$ about here]

Changes in AROM for each intervention are shown in Figure 4. The only significant reductions in stiffness occurred following the standard intervention, but there were regions of increased stiffness following the placebo and control interventions. There were significant differences in within-subject changes by intervention for FE, LF, ROT and TROM ( $F=7.291$, 2.814, 2.970, and 7.929; $\mathrm{DF}=3$ ). The only significant pairwise contrasts for each axis of movement indicated that the differences in AROM were larger for the general intervention than for the other interventions. Changes in regional stiffness following each intervention are

29 interventions $(\mathrm{F}=0.945, \mathrm{DF}=3)$. 
3 The only significant correlation between changes in TROM and mean PA stiffness was for changes in PA stiffness on the symptomatic side following the standard intervention (Table 2). Following the standard intervention, the only individual axis of AROM that demonstrated a significant correlation with mean PA stiffness was LF $(R=-0.459, p=0.024)$. Three out of the four regions of stiffness (8 - 14 N, $14-18 \mathrm{~N}$ and $18-25 \mathrm{~N}$ ) demonstrated significant correlations with TROM $(\mathrm{R}=-0.466,-0.528$ and $-0.628 ; p=0.022,0.010$ and 0.002$)$.

[Insert Table 2 about here]

\section{Discussion}

13 Movement diagrams, single values of displacement and linear approximations of stiffness

14 have been previously advocated to describe PA movements. The current study used a 15 continuous assessment of stiffness to assess PA movements. This method using continuous 16 values and considering derivatives of the raw data is consistent with methods of analysis

17 advocated for other time series data ${ }^{29}$. The current study aimed to determine whether 18 differences in AROM and PA stiffness following treatment by PA movements to the cervical 19 spine were dependent on the treated location and if there is a relation between improvement in AROM and reduction in PA stiffness.

The expected increase in AROM following the standard intervention was not detected although an increase in AROM did occur following the general intervention. The general intervention was intended to be similar to a typical clinical treatment and the increases in AROM that were found were consistent with other studies of immediate changes in AROM following manual therapy treatment ${ }^{6,35,36}$. The general intervention included treatment to several areas of the neck and upper back and the improvement in AROM following the general treatment but not the standard intervention highlights one of the difficulties with manual therapy research. When interventions are pre-selected or pre-specified as for the standard intervention in the current study and as is often required by research designs, the treatment may not be as effective as treatments applied in clinical practice ${ }^{24}$. Alternatively, 
1 the general intervention always being performed last may have influenced the relative size of

2 the increase in AROM following this intervention.

The decrease in PA stiffness following the standard treatment that would have been expected according to the manual therapy paradigm only occurred at forces from 4-8 $\mathrm{N}$ and 14-18 $\mathrm{N}$. Differences in PA stiffness were not detected following treatment in two previous studies of the lumbar spine. One study involved asymptomatic subjects ${ }^{1}$ and the other involving

8 symptomatic subjects did not detect differences in PA stiffness following treatment in spite of 9 an improvement in pain ${ }^{10}$. In both studies however the investigators only considered single 10 values of displacement or stiffness.

The hypothesis of a relation between increases in AROM and decreases in PA stiffness at the symptomatic location following the standard intervention was supported by the results. To our knowledge, this is the first demonstration of a relation between PA stiffness and impairments in the cervical spine. Latimer et al. ${ }^{19}$ found decreased stiffness at forces over $30 \mathrm{~N}$ and an increased PA displacement up to a force of $30 \mathrm{~N}$ in a group of patients with low back pain when their pain had resolved by $80 \%$. Kulig et al. ${ }^{17}$ on the other hand found increased PA mobility in a group of young adults with low back pain compared to asymptomatic controls, but this comparison of group differences would not distinguish between differences related to pre-existing or pre-disposing factors and those related to the source of the patient's impairments.

The finding that relations between changes in PA stiffness and AROM only occurred following treatment to the symptomatic location and that similar changes in PA stiffness did not occur at an untreated location suggests a localization of the effects of treatment by PA movements. Similarly, Chiradejnant et al. ${ }^{8}$ found that improvement in pain following treatment by PA movements was greater when the treated location had been previously selected as a likely source of impairments.

One of the main aspects of PA stiffness investigated previously is stiffness at forces near the 'end of range' ${ }^{23}$. The findings in the current study of a relation between changes in AROM 
and PA stiffness at all force regions above $8 \mathrm{~N}$ are similar to the results of our previous study where differences in PA stiffness were found between tender and less tender locations of the cervical spine for all forces above $11.5 \mathrm{~N}^{38}$. The changes that were found in stiffness included force levels much lower than forces of up to $100 \mathrm{~N}$ that have been reportedly used by therapists to produce 'end-of-range' PA movements in the cervical spine ${ }^{32}$. The idea that differences in motion palpation can be detected at low forces is supported by previous findings. Marcotte et al. ${ }^{25}$ using motion palpation techniques other than PA movements found that clinicians applying less than five $\mathrm{N}$ of force could accurately detect the location of fused cervical vertebrae. The relatively small sample size in the current study was sufficient to determine that local changes in PA stiffness following manual therapy treatment are related to changes in AROM, but it was not possible to fully characterize these changes and in no case did the changes in PA stiffness account for more than 36\% of the variation in AROM.

Conclusions

15 Following the standard intervention, the expected increase in AROM did not occur, and the decrease in PA stiffness only occurred in response to certain levels of forces. Change in PA stiffness at locations deemed to be symptomatic were only found to be related to change in impairments following manual therapy treatment to the symptomatic location in patients with neck pain for more than 2 weeks. The findings suggest that at symptomatic locations of the cervical spine there is a relation between AROM and PA stiffness at forces as low as eight $\mathrm{N}$

\section{Bibliography}

1. Allison G, Edmonston S, Kiviniemi K, et al. Influence of standardized mobilization on

29 the posteroanterior stiffness of the lumbar spine in asymptomatic subjects. Physiotherapy

30 Research International 2001;6:145-56.

31 2. Allison GT, Edmondston SJ, Roe CP, et al. Influence of load orientation on the

32 posteroanterior stiffness of the lumbar spine. Journal of Manipulative and Physiological

33 Therapeutics 1998;21:534-8. 
13 Bland JM, Altman DG. Measuring agreement in method comparison studies. Statistical

2 Methods in Medical Research 1999;8:135-60.

3 4. Bogduk N, Mercer S. Biomechanics of the cervical spine. I: Normal kinematics. Clinical 4 Biomechanics 2000;15:633-48.

5 5. Caling B, Lee M. Effect of direction of applied mobilization force on the posteroanterior

6 response in the lumbar spine. Journal of Manipulative and Physiological Therapeutics

7 2001;24:71-8.

8 6. Cassidy JD, Lopes AA, Yong-Hing K. The immediate effect of manipulation versus

9 mobilization on pain and range of motion in the cervical spine: a randomized controlled trial. Journal of Manipulative and Physiological Therapeutics 1992;15:570-5.

7. Chansirinukor W, Lee M, Latimer J. Contribution of pelvic rotation to lumbar posteroanterior movement. Manual Therapy 2001;6:242-9.

8. Chiradejnant A, Maher CG, Latimer J, et al. Efficacy of "therapist-selected" versus "randomly selected" mobilisation techniques for the treatment of low back pain: A randomised controlled trial. Australian Journal of Physiotherapy 2003;49:233-41.

9. Edmondston SJ, Allison GT, Gregg CD, et al. Effect of position on the posteroanterior stiffness of the lumbar spine. Manual Therapy 1998;3:21-6.

10. Goodsell M, Lee M, Latimer J. Short-term effects of lumbar posteroanterior mobilization in individuals with low-back pain. Journal of Manipulative and Physiological Therapeutics 2000;23:332-42.

11. Grieve GP. Common vertebral joint problemsed. Melbourne: Churchill Livingstone, 1981.

12. Hahne AJ, Keating JL, Wilson SC. Do within-session changes in pain intensity and range of motion predict between-session changes in patients with low back pain? Australian Journal of Physiotherapy 2004;50:17-23.

13. Hodges P, Kaigle Holm A, Holm S, et al. Intervertebral stiffness of the spine is increased by evoked contraction of transversus abdominis and the diaphragm: in vivo porcine studies.

\section{Spine 2003;28:2594-601.}

14. Jordan K. Assessment of published reliability studies for cervical spine range-of-motion measurement tools. Journal of Manipulative and Physiological Therapeutics 2000;23:180-95.

15. Jull G, Treleaven J, Versace G. Manual examination: is pain provocation a major cue for spinal dysfunction? Australian Journal of Physiotherapy 1994;40:159-65.

16. Kawchuk GN, Kaigle AM, Holm SH, et al. The diagnostic performance of vertebral displacement measurements derived from ultrasonic indentation in an in vivo model of degenerative disc disease. Spine 2001;26:1348-55.

17. Kulig K, Powers CM, Landel RF, et al. Segmental lumbar mobility in individuals with low back pain: in vivo assessment during manual and self-imposed motion using dynamic MRI. BMC Musculoskeletal Disorders 2007;8:8.

18. Lantz CA, Chen J, Buch D. Clinical validity and stability of active and passive cervical range of motion with regard to total and unilateral uniplanar motion. Spine 1999;24:1082-9.

19. Latimer J, Lee M, Adams R, et al. An investigation of the relationship between low back pain and lumbar posteroanterior stiffness. Journal of Manipulative and Physiological Therapeutics 1996;19:587-91.

20. Lee M, Gal J, Herzog W. Biomechanics of manual therapy. In Dvir Z ed. Clinical

Biomechanics. Philadelphia: Churchill Livingstone, 2000:209-37. 
1 21. Magarey M. Examination of the Cervical and Thoracic Spine. In Grant R ed. Physical

Therapy of the Cervical and Thoracic Spine. 3rd ed. New York: Churchill Livingsone, 2002:10537.

22. Maher C, Adams R. A psychophysical evaluation of manual stiffness discrimination. Australian Journal of Physiotherapy 1995;41:161-7.

23. Maitland G. Vertebral Manipulation. 1st ed. London: Butterworths, 1964.

24. Maitland G, Hengeveld E, Banks K, et al. Maitland's Vertebral Manipulation. 7th ed.

Edinburgh; New York: Elsevier/Butterworth Heinemann, 2005.

9 25. Marcotte J, Normand MC, Black P. Measurement of the pressure applied during motion the mechanics of a posterior-anterior mobilisation? Clinical Biomechanics 2001;16:926-9.

27. Nicholson L, Maher C, Adams R, et al. Stiffness properties of the human lumbar spine: a lumped parameter model. Clinical Biomechanics 2001;16:285-92.

28. Petty NJ, Maher C, Latimer J, et al. Manual examination of accessory movements-seeking R1. Manual Therapy 2002;7:39-43.

29. Ramsay JO, Silverman BW. Functional data analysised. New York: Springer, 1997.

30. Shirley D. Manual therapy and tissue stiffness. In Boyling JD, Jull G eds. Grieve's Modern Manual Therapy. Third ed. Sydney: Churchill Livingstone, 2004:381-91.

31. Shirley D, Hodges PW, Eriksson AE, et al. Spinal stiffness changes throughout the respiratory cycle. Journal of Applied Physiology 2003;95:1467-75.

32. Snodgrass SJ, Rivett DA, Robertson VJ. Manual forces applied during cervical mobilization. Journal of Manipulative and Physiological Therapeutics 2007;30:17-25.

33. Squires MC, Latimer J, Adams RD, et al. Indenter head area and testing frequency effects on posteroanterior lumbar stiffness and subjects' rated comfort. Manual Therapy 2001;6:40-7.

34. Sran MM, Khan KM, Zhu Q, et al. Posteroanterior stiffness predicts sagittal plane midthoracic range of motion and three-dimensional flexibility in cadaveric spine segments. Clinical Biomechanics 2005;20:806-12.

35. Tseng YL, Wang WT, Chen WY, et al. Predictors for the immediate responders to cervical manipulation in patients with neck pain. Manual Therapy 2006;11:306-15.

36. Tuttle N. Do changes within a manual therapy treatment session predict between-session changes for patients with cervical spine pain? Australian Journal of Physiotherapy 2005;51:43-8. 37. Tuttle N, Barrett R, Laakso L. Postero-anterior movements of the cervical spine: Repeatability of force displacement curves. Manual Therapy 2007.

38. Tuttle N, Barrett R, Laakso L. Posteroanterior movements in tender and less tender locations of the cervical spine. Manual Therapy 2007.

39. Tuttle N, Laakso L, Barrett R. Change in impairments in the first two treatments predicts outcome in impairments, but not in activity limitations, in subacute neck pain: an observational study. Australian Journal of Physiotherapy 2006;52:281-5. 
PA Stiffness
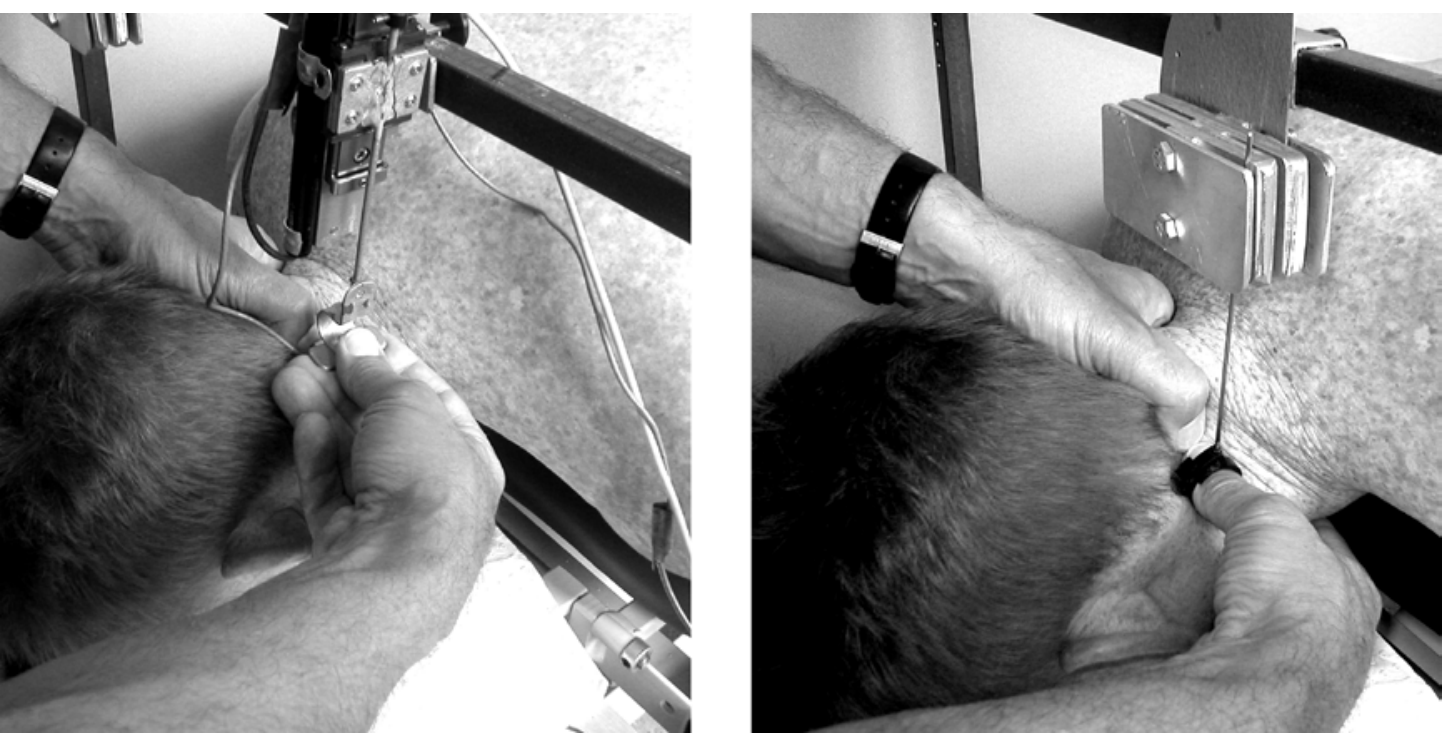

2 Figure 1

3 PA movements using the PMAD and with gating mechanism used during treatment in the current

4 study to ensure a consistent location. The patient is prone on a modified treatment bed and a PA

5 force is applied unilaterally over the articular pillar. For the PMAD shown on the left a load cell

6 is between the therapist's hand and the indentor that contacts the patient's neck. As shown on the

7 right, a gating mechanism attached to the same frame as the PMAD was used during assessment

8 and treatment to ensure a consistent location of PA movements both with repeated movements

9 and with the PMAD.

10

11

12
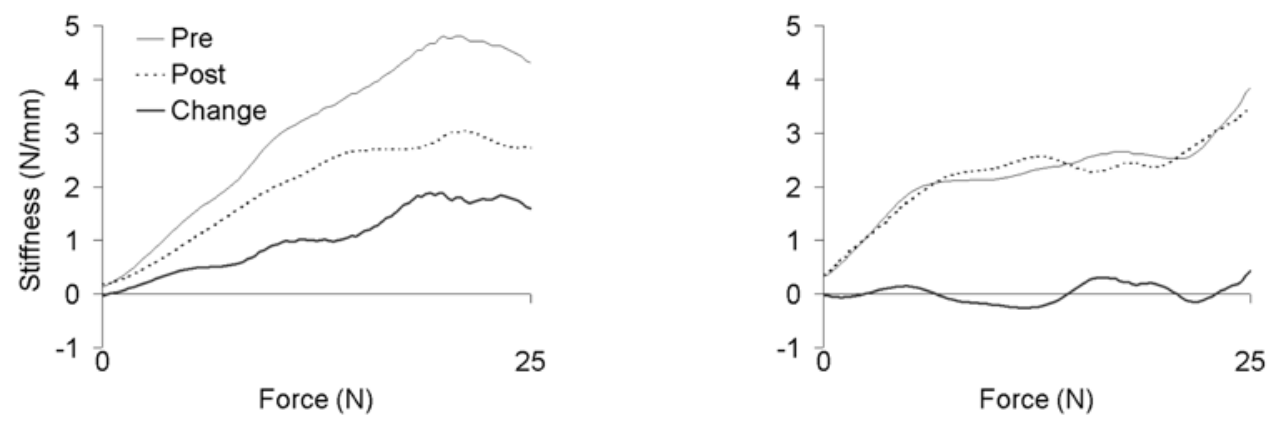

13

14 Figure 2 
1 Representative data from the symptomatic locations of two subjects showing stiffness force

2 curves from pre-intervention and post-intervention PAs and changes in stiffness following the 3 standard intervention.
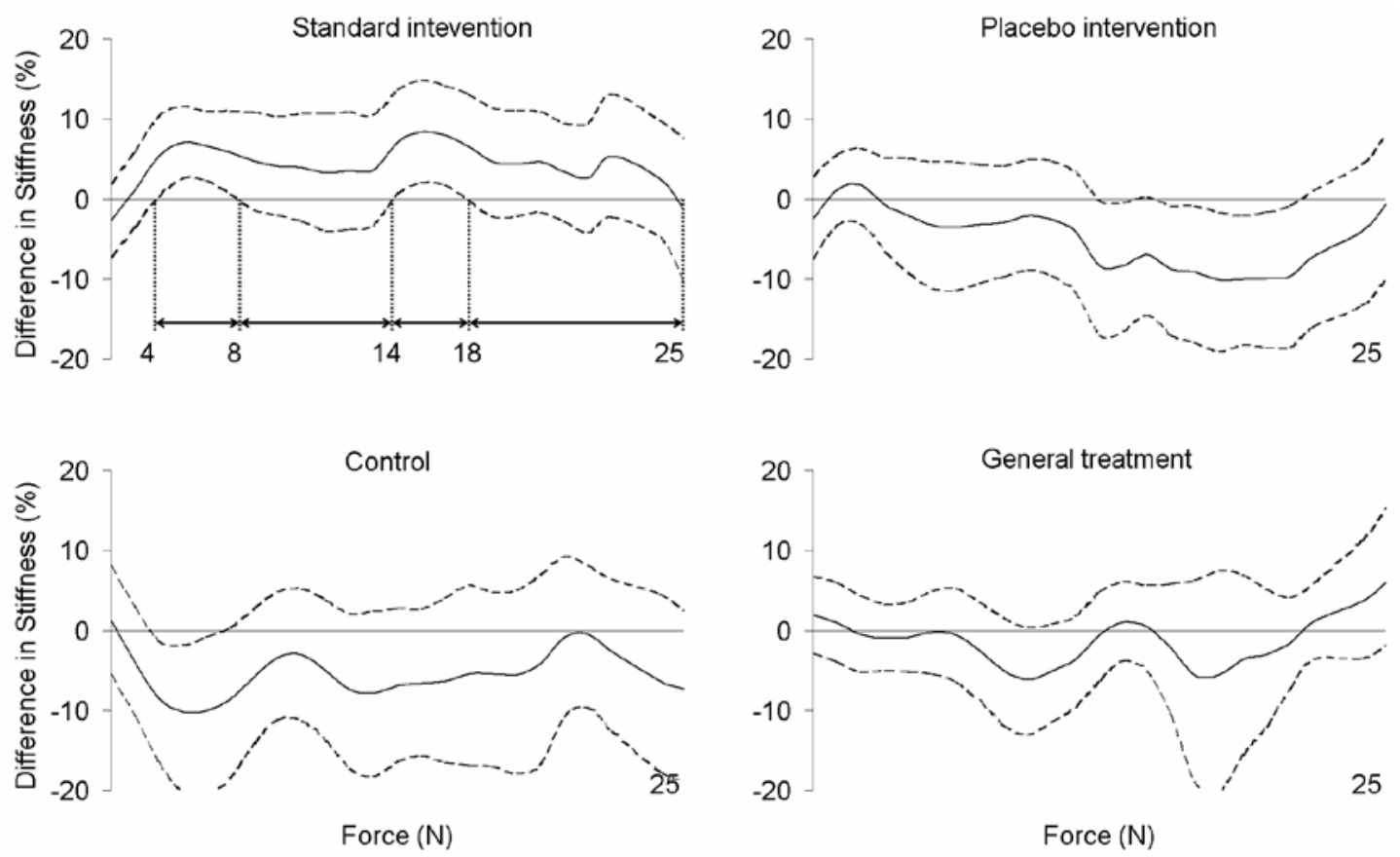

9 Figure 3

10 Average percentage change in stiffness of the entire sample on the symptomatic side following

11 each intervention. The solid lines represent the mean percentage change in PA stiffness and the

12 dashed lines the 90\% SCBs. Regions of stiffness where the SCBs include and do not include zero

13 for the standard intervention are used in further analysis and are indicated on the graph at the top

14 left.

15

16

17

18 
PA Stiffness

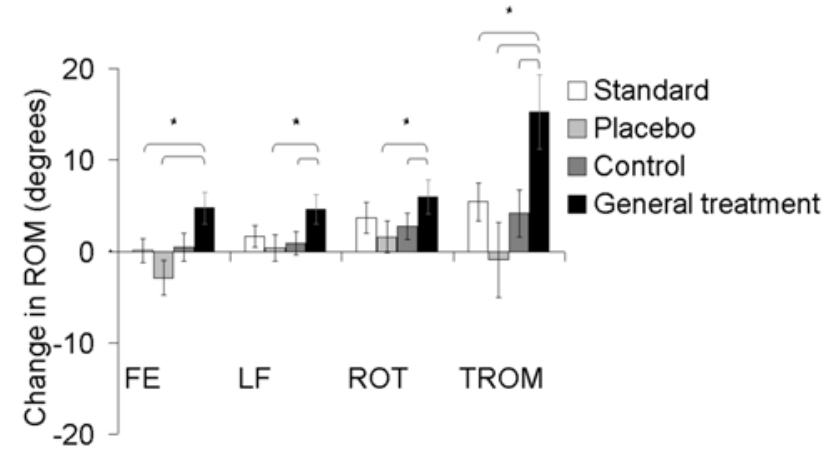

$3 \quad$ Figure 4

4 Changes in AROM by intervention. Error bars represent one standard error of the mean. Asterisk

$5 \quad(*)$ indicates significant within subject differences across interventions.

6
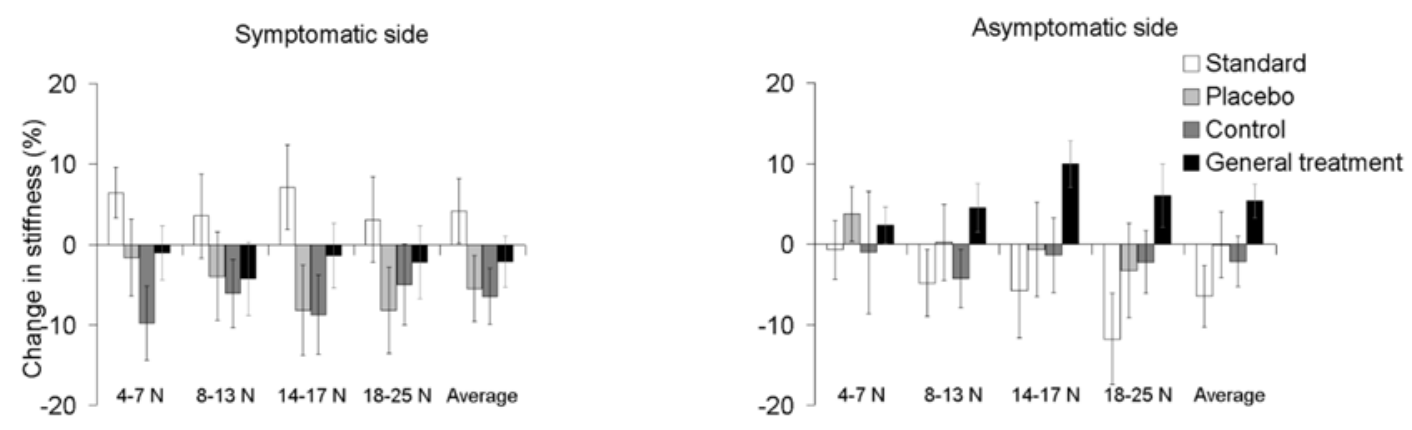

8

$9 \quad$ Figure 5

10 Percentage changes in regional stiffness of the symptomatic and asymptomatic locations

11 following the standard treatment (PA movements to the symptomatic location). Error bars

12 represent one standard error of the mean.

13

14

15 